\title{
Based on local structural similarity image denoising algorithm
}

\author{
Zehua Zhou Weitang Zhang \\ Department of Electronics \& Electrical Engineering \\ Hefei University \\ Hefei, China \\ E-mail: zhouzh@hfuu.edu.cn
}

\begin{abstract}
The image is a kind of important information source, through the image processing can help people understand the connotation of the information. However, the image in the process of generation and transmission by all kinds of the noise, the information processing, transfer and storage caused tremendous influence. So the image denoising always all is the computer image processing and computer in the vision of a research focus. Proposed an algorithm of image denoising based on the local structural similarity. Which utilizes the redundant information, and by establishing a similar function to the search area calculation of point and to pixels similarity of weights, and then to the search area at the weighted, obtained the last to pixels gray value varies. This algorithm in texture, of the edge information denoising ways than the current many denoising algorithm are excellent.
\end{abstract}

Keywords- local; structural similarity; image denoising

\section{INTRODUCTION}

The image is one of the important sources of information society access to information. Useful in the real world image signal by the image sensor acquisition, quantization, coding, transmission, recovery process, there are a lot of factors that affect image quality. Therefore, prior to the use of the image carrying, are generally required to undergo a rigorous pretreatment, such as denoising, quantization, and compression. Noise pollution is a direct impact on the image edge detection, feature extraction, image segmentation, pattern recognition and processing, so that people have to be explored from various angles in order to improve the quality of the image. Therefore, using appropriate methods to try to eliminate the noise is a very important preprocessing step in image processing.

Between the adjacent pixels in the image processing, most of them have a high correlation, that is, most of the pixels in an image gray is not very different ${ }^{[1]}$. Because of the existence of this gray-scale correlation, the general image of the energy is mainly concentrated in the low frequency region, only the details of the image part of the energy only in high-frequency region. Often because of the noise appears in the image digitization and transmission, this part of the interference information is mainly concentrated in the highfrequency region. Therefore, the general approach is to eliminate noise attenuation of high frequency components, or low-pass filter. But with the same time the negative impact of the details of the image a certain attenuation of the visual effects on images blurred than before treatment. A better denoising method is not only to eliminate the impact of noise on the images without blur image details ${ }^{[2]}$. According to the noise spectral distribution law and statistical features, as well as the characteristics of the image, there has been a wide variety of denoising method. Classical denoising methods: synthetic method for the synthesis of the spatial domain, frequency domain and the optimal linear synthesis method ${ }^{[3]}$ were corresponding to the many application methods: such as the mean filter, median filter, low pass filter, Wiener filter, the minimum distortion method ${ }^{[4]}$. These methods are widely used to promote the great development of the digital signal processing significantly improves the image quality. In recent years, there are many denoising algorithms have been proposed, buades et al. proposed NL-Means algorithm take full advantage of the similarity of the local structure of images, with good denoising effect ${ }^{[5-7]}$.

\section{NOISE IMAGE MODEL AND NOISE CHARACTERISTICS}

\section{A. Noisy model}

The reality of the digital image digitization and transmission process, often by the imaging device and the external environmental noise impact and become noisy images. Remove or reduce the noise to obtain a digital image is called image denoising. Before denoising, we need to establish a model of a noisy image. For simplicity, we study the following additive noise model, the noisy image only the original image superimposed on a random noise formation:

$$
g(x, y)=f(x, y)+v(x, y)
$$

$f(x, y)$ said image, $v(x, y)$ is the noise, noisy image is denoted by $g(x, y)$.

\section{B. Noise characteristics}

Before this including noise model, we need to look at the characteristics of the noise, and often affects the image quality of the noise source can be divided into three categories. People made a lot of research on the cause of noise generation and the corresponding model:

- Electronic noise. Electronic noise due to electronic random thermal motion in the resistive device is the easiest of the three models, the commonly used zeromean Gaussian white noise as its model, it can be used and the standard deviation to fully characterize. 
- $\quad$ Photoelectron noise. Caused by the statistical nature of light and image sensors, photoelectric conversion process, commonly used in the case of weak light with a Poisson distribution of random variables as the model of the photoelectric noise in the light stronger, the Poisson distribution tend to be easier to describe the Gaussian distribution.

- $\quad$ Sensitive film particle noise. Because only a portion of the light-sensitive particles in the exposure process has been exposed, while the rest of the unexposed film density changes determined by the change of the particles after the exposure intensity, while the operator exposure the distribution of the particles present a randomness. In most cases, the grain noise is available in white Gaussian noise as a valid model.

It can be seen by the above analysis, the most common image noise are available with zero mean and variance of different Gaussian white noise as its model, thus the sake of simplicity and generalization, we use a zero mean Gaussian white noise as a noise source.

\section{LOCAL STRUCTURAL SIMILARITY IMAGE DENOISING ALGORITHM}

\section{A. Local structural similarity image denoising algorithm principle}

The basic idea: the current pixel value from the image and its structure is similar to the pixel-weighted average. Weights for each pixel, using it as the center image subblock (usually take $7 \times 7$ ) with the Gaussian weighted Euclidean distance between the center of the sub-block to the current pixel to calculate the weights set to this distance from the negative exponential function value. The benefits of doing so is in estimating the current pixel value, with it on the local structure similar to the pixel power heavy, while the structure is similar noise superimposed on the pixel is random, and thus by the weighted mean can effectively remove the noise.

For a discrete noise images:

$$
v(i)=\{v(i), i \in I\}
$$

A pixel $\mathrm{k}$, we require that $N_{k}$ is the rectangular neighborhood which is as a center of $\mathrm{k}$. So, the image of $\mathrm{v}$, the pixel $\mathrm{i}$ and pixel $\mathrm{j}$ Gaussian weighted Euclidean distance is expressed as:

$$
\left\|v\left(N_{i}\right)-v\left(N_{j}\right)\right\|_{2}^{2}
$$

So you can get a description of the weight of pixel $\mathrm{i}$ and pixel $\mathrm{j}$ the degree of similarity:

$$
w(i, j)=\frac{1}{Z(i)} \exp \left(-\frac{\left\|v\left(N_{i}\right)-v\left(N_{j}\right)\right\|_{2}^{2}}{h^{2}}\right)
$$

Among them,

$$
z(i)=\sum \exp \left(-\frac{\left\|v\left(N_{i}\right)-v\left(N_{j}\right)\right\|_{2}^{2}}{h^{2}}\right)
$$

$Z(i)$ is the right value of the normalization coefficient, and the $h$ is the image of the filter parameters. The parameter $\mathrm{h}$ to control the size of the weights to control the degree of smoothing noise attenuation control of the power function, if relatively small, the attenuation effect of the power function more significant details of the extent of any reservations, so it will keep the details of the image itself. At the same time, the weights $w(i, j)$ also satisfy the following conditions: $0 \leq w(i, j) \leq 1$ and $\sum w(i, j)=1$.

Pixel $\mathrm{i}$ and pixel $\mathrm{j}$ similar extent dependent on the degree of similarity in the rectangular neighborhood of $v\left(N_{i}\right)$ and $v\left(N_{j}\right)$, the greater the weight value, the rectangular neighborhood of the image will be similar.

Fig. 1 is the degree of similarity of examples of the image. Pixel p and pixel q1 have similar rectangular neighborhood, the lower rectangular neighborhood of pixel p and pixel q2. Therefore, we calculate the value of denoising the right will find the value of $w(p, q 1)$ is greater than the value of $w(p, q 2)$, that is, the pixel q1 denoising the right value is much larger than the pixel q2.

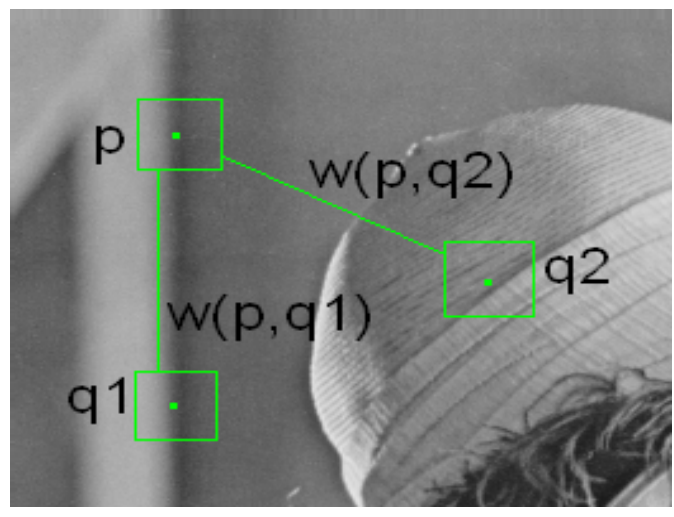

Figure 1. The degree of similarity

Final denoising results are:

$$
N L(v)(i)=\sum_{j \in I} w(i, j) v(j)
$$


B. Local structure similarity algorithm (LSS) with other denoising algorithms

Mean filtering, median filtering, Wiener filtering and the local structure similarity algorithm for image denoising the simulation, and the corresponding simulation results are analyzed in this paper, the simulation results shown in Fig.2 to 7.

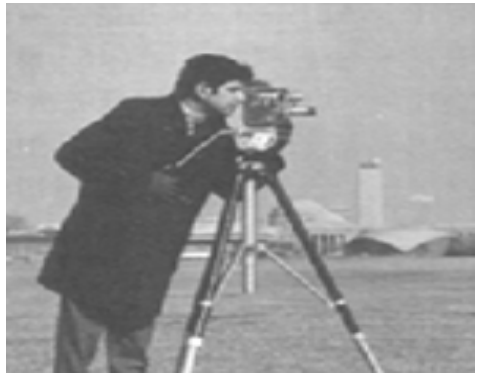

Figure 2. original image

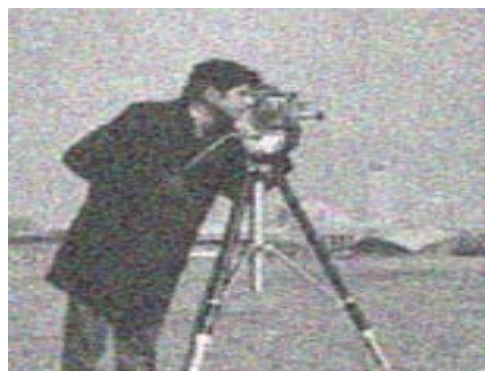

Figure 3. noise image

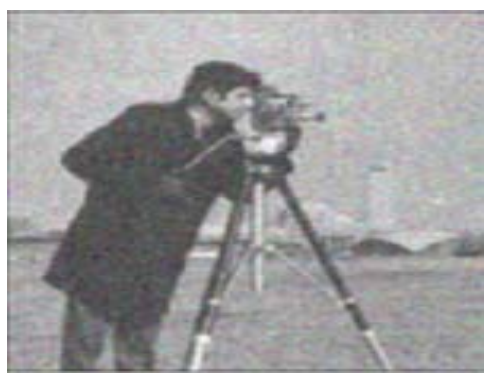

Figure 4. Mean filter

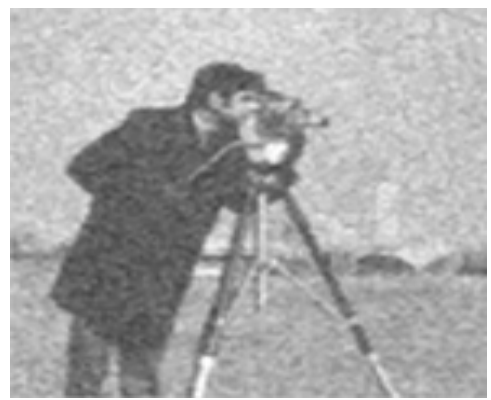

Figure 5. Median filter

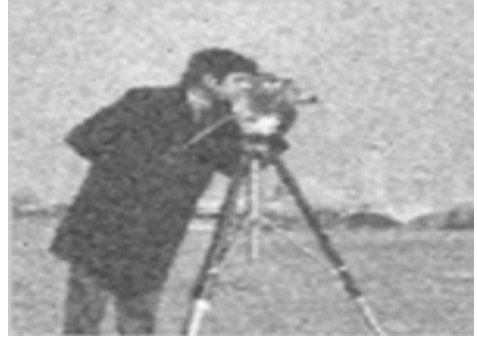

Figure 6. Wiener filter

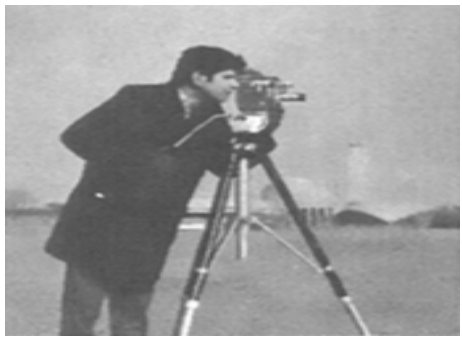

Figure 7. LSS algorithm

From the simulation results can be seen, the neighborhood averaging method is easy to use, apply to the elimination of grain noise in the image, but it should be noted that this method is both smooth the image signal, while making the details of the part of the image blurred. Fig. 4 can be seen of the neighborhood average weaken the image of the edge of the image become somewhat blurred. Mean filtering of Gaussian noise suppression is good, bad in order to improve the contrast of details of the mean filter fuzzy region boundaries defects commonly used threshold to suppress the fine texture of the salt and pepper noise and the protection of the weighting method to improve the image of the fuzzy boundaries, with select the average adaptive technology to maintain the boundaries of the image. As for Gaussian noise, shown in Fig.5, although there are some denoising, but ineffective. Therefore, the median filter operation is simple, easy to implement, and can better protect the border, but sometimes lose the image of the thin and small area. Using the window size can be a great influence on the filtering effect, the greater the window, the better denoising effect, but at the cost the greater the degree of ambiguity. Wiener filtering of white Gaussian noise image filtering with the neighborhood average, good filtering effect, it has a better selectivity than the linear filter, better to save the image edges and high frequency details. Although the Wiener filter in most cases can be satisfied with the results, especially the image containing Gaussian noise. It can not be used for non-stationary random noise process, applied for the vector case is not convenient. Therefore, the Wiener filter in practical problems is limited. Compared to the above algorithm, the effect of local structure similarity algorithm on Gaussian white noise image filtering, image details can be better preserved. Into the local structure similarity algorithm filtering the image is not only removed a lot of noise points, 
but also in the edges and lines become clearer than ever before.

\section{CONCLUSIONS}

The traditional denoising method in denoising the same time, details of the image blurred. Contemporary highly information-oriented society, the role of graphics and images in the dissemination of information is growing. Therefore, to eliminate the noise in the image acquisition and transmission process to ensure that the image of an important part of the pollution of the smallest has become the field of digital image processing. In this paper, the denoising algorithm based on local structural similarity has a structure similar to the region have a greater right to have lesser rights or zero structure similar to the area, therefore, the ability to retain the structural information in the image. The visual quality of denoised image has been greatly improved. The computational complexity of the proposed algorithm is higher than some of the traditional algorithm. Local structural similarity denoising will be one of the important aspects of denoising.

\section{ACKNOWLEDGMENT}

This paper supported by Natural Science Foundation of Anhui Province in University（KJ2011B136）,thanks.

\section{REFERENCES}

[1] STACK JL, and MURTAGH F, "Gray and color image constrast enhancement by the curvelet transform," IEEE Trans on Image Processing.America,vol.12,pp.706- 716,june 2003.

[2] CAITT,and SILVERMANBW, "Incorporating information on Neighbouring Coefficients into wavelet estimation,"The Indian Journal of Statistics.Indian,vol 63,pp.127-148,february 2001.

[3] YANG Qun_sheng, CHEN Ming, and YU Ying_lin, “The Random Noise Removal of the Corrupted Image Based on Fuzzy Technique ,"Journal of South China University of Technology(Natural Science Edition).China,vol.28,pp.82-87,august 2000.

[4] L.K.Shark, and C.Yu, "Denoising by optimal fuzzy thresholding in wavelet domain,”IEEE Electronics letters.America,vol 36,pp.581582,june 2000.

[5] Buade A, "Morel JM.A non-local algorithm for image denoising,"Proceedings Of IEEE Computer Society Conference on Computer Vision and Pattern Recognition.San Diego,2005,pp.60-65.

[6] M ahmoud iM, and Sapiro G1, "Fast image and video denoising via non local means of similar neighborhoods,”IEEE Signal Processing Letters.America,vol.12,pp839-8421,december 2005.

[7] WANG Zhi-ming, and ZHANG Li, “An Adaptive Fast Non Local Image Denoising Algorithm,"Journal of Image and Graphics. China,vol.14,pp.669-675,april 